\title{
Histone acetyltransferase inhibitors: where art thou?
}

\author{
"...is catalytic activity of histone acetyltransferase an \\ undruggable mechanism?"
}

\section{First draft submitted: 19 July 2016; Accepted for publication: 27 July 2016; Published online: 24 August 2016}

Keywords: histone acetyltransferase inhibitors • lysine acetyltransferase inhibitors - small-molecule epigenetic modulators

As many readers of this journal will be aware, epigenetic modifications do not relate directly to the genetic code, but rather to changes in the structure of DNA and associated histone proteins. Despite originating more than half a century ago [1] it is only in relatively recent times that the field of epigenetics has garnered broad and high level interest from the standpoint of therapeutic intervention. Many medicinal chemists would argue that such interest is higher now than it has ever been before.

It is not hard to see why many in drug discovery are particularly excited by the prospect of small-molecule epigenetic modulators because epigenetic modifications are associated with alteration of chromatin and transcriptional action, affecting DNA conformation with either activation or repression of genes as a direct consequence [2]. Given that cancer is essentially a disease of dysregulated gene expression, involving activation of oncogenes and silencing of tumor suppressors, that epigenetic modification could result in cancer is a 'clear and present danger'. However, the potential of epigenetic therapy extends well beyond the realm of cancer and further, as the epigenetic process is relevant to the development and proper functioning of the myriad of pathogens or pests that afflict humanity, the potential verges on limitless.

Epigenetic-regulating proteins comprise a broad church, including 'writers' (acetyltransferases, methyltransferases), 'readers' (bromodomains, tudor domains, chromodomains, PHD finger domains) and 'erasers' (deacetylases, demethylases), which all perform distinctly different functions.

Some of these denominations are already targeted by successfully marketed drugs, while others are progressing through clinical and preclinical development. Well represented in this pipeline as drugs, drug candidates or as useful tool compounds are inhibitors of 'writers', including DNA methyltransferases and more recently lysine and arginine methyltransferases, 'readers' such as bromodomains, and 'erasers' such as histone deacetylases. For example, there are more than 18 clinical trials underway for BET bromodomain inhibitors [3].

In this special issue of Future Medicinal Chemistry some of the excellent developments in these areas are showcased, with a notable exception: inhibitors of lysine acetyltransferases (KATs) that target the catalytic machinery. This is because, intriguingly, high quality inhibitors with such proven mechanisms of action appear to be essentially absent in the scientific literature. Why is this so? This observation merits further comment.

\section{Inhibiting the HAT in the KAT}

While there are subclass details beyond the scope of this commentary but are listed elsewhere [4], KATs are typically grouped into three broad families, these being the p300/CBP, the Gcn5 related $N$-acetyl-

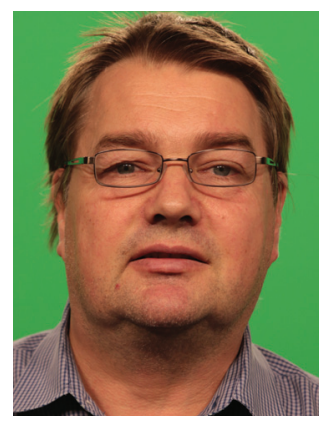

Jonathan B Baell

Author for correspondence:

Medicinal Chemistry, Monash Institute of Pharmaceutical Sciences, Monash University, 381 Royal Parade, Parkville, Victoria 3052, Australia jonathan.baell@monash.edu

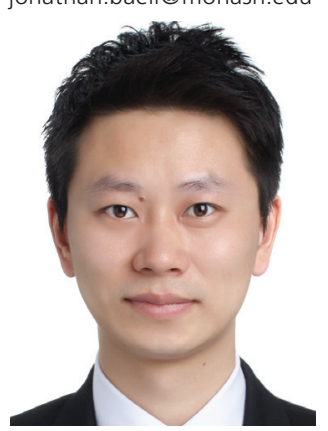

Wenjun Miao

School of Pharmaceutical Science, Nanjing Tech University, Nanjing, 211816, China 
transferase (GNAT) and the MYST family of KATs. Including species other than Homo sapiens, the GNAT family comprises about seven KATs, the p300/CBP family comprises just themselves and the MYST family comprises about eight KATS and indeed represents about a third of all KATs in the human genome [4]. The MYST family is so-named because of a common MYST homology region within the catalytic domain.

While KATs acetylate lysine residues in a variety of contexts, it is their dominating role in regulating gene expression and chromatin structure by post-translational covalent modification of histone lysine residues in chromatin, which has attracted most interest. These histone acetyltransferases (HATs) acetylate histones in bound chromatin structure, neutralizing the attractive forces between positively charged lysines and negatively charged DNA, resulting in loosening of the previously compact structure. This increases access to DNA by transcriptional machinery, as well as providing opportunities for 'readers' to act through binding the newly acetylated lysine-containing sequences.

\section{"Knowledge of enzyme mechanism also allows for rational approaches to ligand design and discovery."}

The biological consequences of dysregulation can be profound. A case in point is provided by the MYST HAT known as MOZ (also as MYST3 or KAT6A), which is associated with dire hematopoietic malignancies. Indeed, genetic reduction of $\mathrm{Moz}$ confers remarkable survival outcomes in murine models of certain lymphomas. For example, in the E $\mu-\mathrm{Myc}$ model of aggressive pre-B/B-cell lymphoma, loss of just one allele of Moz increased the median survival of mice 3.9-fold [5]. These genetic studies suggest that pharmacological inhibition of MOZ, and consequent decreased lysine acetylation levels, may be useful for treating certain lymphomas and leukemias. This is somewhat counterintuitive when considering the marketed HDAC inhibitor vorinostat, which is being used to increase acetylation in cutaneous T-cell lymphoma treatment [6]. The apparent paradoxes of complex biology!

Understandably, the search for small-molecule HAT inhibitors has been intense and yet there is very little evidence for useful compounds having been developed and clinically progressed. One contributing reason for this might be that high-throughput screening (HTS) campaigns against HATs have resulted in very noisy data, making identification of the few real hits among the false positives very difficult. For example, triage of cell-based screening data of more than 600,000 compounds eventually yielded a compound known as L002 with in vivo tumor growth reduction corre- lated to HAT inhibition [7]. However, as is the case for many reported KAT inhibitors, we would strongly identify this quinoid-type molecule as a PAIN [8-10]. Pan-assay interference compounds (PAINS) are generally reactive compounds likely to mislead researchers into nonprogressable drug development and are commonly mistakenly reported as useful tools, unearthed not just through HTS but also in silico screening $[9,10]$. While L002 was synthetic in origin, screens have also unearthed natural product PAINS [8,11] such as the catechol-containing natural product, garcinol. These, too, are unprogressable compounds from a target-centric perspective. Perhaps the most convincing inhibitor of any human HAT has been a synthetic compound known as C646 and reported to be a p300/CBP inhibitor, but even in this case, its utility is being questioned on the basis of recent data that points to concerning chemical reactivity [12].

Mechanistic knowledge can usefully inform on inhibitor discovery approaches. The endogenous acetylation reagent used by all HATs is the cofactor AcCoA and all HATs bind AcCoA as part of this mechanism. The acetyl group of AcCoA is then transferred, either directly or indirectly, to cobound histone (although it is only for GNAT and MYST HATs that significant histone binding appears to be required during this process), producing $\mathrm{CoA}$, which now has a free thiol group [13].

On the basis of this knowledge, Dahlin et al. undertook HTS against Rtt109, a fungal HAT, using a thiolsensitive fluorescent probe to detect CoA concentration as means to gauge degree of HAT inhibition asserted by test compound. However, excellent postmortem forensic investigation determined that this HTS resulted in detection of a large number of assay artifacts, these being mostly small molecules that had thiol reactivity [14]. Prior to that report, we had already run into different, yet related, problems. In 2010 we undertook HTS against MOZ, using an antiacetyllysine antibody to detect acetylated histone concentration as means to gauge the degree of HAT inhibition asserted by each test compound. We found the screening hits to be almost exclusively artifactual, dominated by acetamides that fooled the antibody into recognizing them as acetylated lysine [15].

Knowledge of enzyme mechanism also allows for rational approaches to ligand design and discovery. In the case of HATs, this approach has led to the successful identification of bisubstrate analogs, combining AcCoA mimics with histone segment mimics. Although such ligands can be regarded as genuine biochemical inhibitors, clearly their structures could not be considered to be drug-like, and cell permeability is unlikely [16]. 
This mechanism of acetyl transfer is important in the context of small-molecule drug discovery because it may begin to explain why good inhibitors of HAT catalytic machinery have been hard to find. As might be expected for any ternary complex involving molecular entities such as enzyme, AcCoA, or histone peptide, structural information confirms that an expansive network of electrostatic interactions appears to be important in catalytic sites [4]. In particular, the pyrophosphate group of AcCoA makes several electrostatic contacts that are likely to be important. In this context, it is readily understood how it might be hard to find drug-like small molecules that compete with AcCoA binding.

\section{Future directions for inhibitors of HAT}

The trials and tribulations underpinning HAT inhibitor drug discovery can be partnered with a variety of reasons that might account for the paucity of quality small-molecule inhibitors that directly inhibit HAT catalytic machinery. The disparity between the high volume of poor quality compounds and near absence of high quality compounds begs the question: "Is catalytic activity of HATS an undruggable mechanism?"

A recent patent from Abbvie workers is suggestive of an answer in the negative. This extensive patent discloses substituted hydantoin derivatives as potent inhibitors of p300/CBP HAT activity [17]. As is the want of patent literature, data are not presented in a fashion that allows for rigorous prosecution from a scientific perspective. Nevertheless, novel and drug-like

\section{References}

1 Virani S, Colacino JA, Kim JH, Rozek LS. Cancer epigenetics: a brief review. ILAR J. 53(3-4), 359-369 (2012).

2 Farria A, Li W, Dent SY. KATs in cancer: functions and therapies. Oncogene 34(38), 4901-4913 (2015).

3 Huston A, Arrowsmith CH, Knapp S, Schapira M. Probing the epigenome. Nat. Chem. Biol. 11(8), 542-545 (2015).

4 Simon RP, Robaa D, Alhalabi Z, Sippl W, Jung M. KATching-up on small molecule modulators of lysine acetyltransferases. J. Med. Chem. 59(4), 1249-1270 (2016).

5 Sheikh BN, Lee SC, El-Saafin F et al. MOZ regulates B-cell progenitors and, consequently, Moz haploinsufficiency dramatically retards MYC-induced lymphoma development. Blood 125(12), 1910-1921 (2015).

6 Mann BS, Johnson JR, Cohen MH, Justice R, Pazdur R. FDA approval summary: vorinostat for treatment of advanced primary cutaneous T-cell lymphoma. Oncologist 12(10), 1247-1252 (2007).

7 Yang H, Pinello CE, Luo J et al. Small-molecule inhibitors of acetyltransferase p300 identified by high-throughput
HAT inhibitors with mechanism-based cellular activity are convincingly portrayed. This corporate effort may not yet, or even ever, manifest in a peer-reviewed publication but ironically is all the more convincing as a result: pharmaceutical companies succeed on the back of valuable discoveries not scientific publications.

In an intriguing twist, it appears that this HAT inhibitor chemotype has also been independently discovered by academic researchers, who in 2013 reported the HTS-discovery of a similar compound as a potent inhibitor of the fungal HAT, Rtt109 [18]. With a surprisingly good SAR set available 'by catalogue', this group provides a package of quality data that all but verifies this chemotype as a legitimate HAT inhibitor. The kicker? These hydantoins appear to be uncompetitive inhibitors: they do not compete with either AcCoA or histone substrate but rather, they likely bind to and stabilize enzyme-substrate complexes. So is catalytic activity of HATS an undruggable mechanism? When it comes to direct, small-molecule inhibitors, it appears that the jury is still out.

\section{Financial \& competing interests disclosure}

The National Health and Medical Research Council of Australia (NHMRC) is thanked for Fellowship support for JB Baell (Senior Research Fellowship \#1020411). The authors have no other relevant affiliations or financial involvement with any organization or entity with a financial interest in or financial conflict with the subject matter or materials discussed in the manuscript apart from those disclosed.

No writing assistance was utilized in the production of this manuscript.

screening are potent anticancer agents. Mol. Cancer Ther. 12(5), 610-620 (2013).

8 Baell J, Walters MA. Chemistry: chemical con artists foil drug discovery. Nature 513(7519), 481-483 (2014).

9 Baell JB. Observations on screening-based research and some concerning trends in the literature. Future Med. Chem. 2(10), 1529-1546 (2010).

10 Baell JB, Holloway GA. New substructure filters for removal of pan assay interference compounds (pains) from screening libraries and for their exclusion in bioassays. J. Med. Chem. 53(7), 2719-2740 (2010).

11 Baell JB. Feeling nature's PAINS: natural products, natural product drugs, and pan assay interference compounds (PAINS). J. Nat. Prod. 79(3), 616-628 (2016).

12 Shrimp JH, Sorum AW, Garlick JM, Guasch L, Nicklaus MC, Meier JL. Characterizing the covalent targets of a small molecule inhibitor of the lysine acetyltransferase P300. ACS Med. Chem. Lett. 7(2), 151-155 (2016).

13 Cortopassi WA, Kumar K, Duarte F, Pimentel AS, Paton RS. Mechanisms of histone lysine-modifying enzymes: a computational perspective on the role of the protein environment. J. Mol. Graph. Model. 67, 69-84 (2016). 
14 Dahlin JL, Nissink JWM, Strasser JM et al. PAINS in the Assay: chemical mechanisms of assay interference and promiscuous enzymatic inhibition observed during a sulfhydryl-scavenging HTS. J. Med. Chem. 58(5), 2091-2113 (2015).

15 Falk H, Connor T, Yang H et al. An efficient highthroughput screening method for MYST family acetyltransferases, a new class of epigenetic drug targets. Anal. Chem. 16(10), 1196-1205 (2011).
16 Lu D. Epigenetic modification enzymes: catalytic mechanisms and inhibitors. Acta Pharmaceutica Sinica B 3(3), 141-149 (2013).

17 ABBVIE, INC.: WO2016/044777A1 (2016).

18 Lopes da Rosa J, Bajaj V, Spoonamore J, Kaufman PD. A small molecule inhibitor of fungal histone acetyltransferase Rtt109. Bioorg. Med. Chem. Lett. 23(10), 2853-2859 (2013). 